\title{
Triatomine bugs, their microbiota and Trypanosoma cruzi: asymmetric responses of bacteria to an infected blood meal
}

\author{
Sebastián Díaz $z^{1}$, Bianca Villavicencio ${ }^{2}$, Nathália Correia ${ }^{3}$, Jane Costa $^{3}$ and Karen L. Haag ${ }^{1,24^{*}}$
}

\begin{abstract}
Background: Triatomine bugs (Hemiptera: Reduviidae) are vectors of the flagellate Trypanosoma cruzi, the causative agent of Chagas disease. The study of triatomine gut microbiota has gained relevance in the last years due to its possible role in vector competence and prospective use in control strategies. The objective of this study is to examine changes in the gut microbiota composition of triatomines in response to a T. cruzi-infected blood meal and identifying key factors determining those changes.

Results: We sampled colony-reared individuals from six triatomine vectors (Panstrongylus megistus, Rhodnius prolixus, Triatoma brasiliensis, T. infestans, T. juazeirensis and T. sherlocki) comparing experimentally T. cruzi strain 0354challenged and non-challenged insects. The microbiota of gut and gonad tissues was characterized using high throughput sequencing of region V3-V4 of bacterial 16S rRNA gene. The triatomine microbiota had a low intraindividual diversity, and a high inter-individual variation within the same host species. Arsenophonous appeared as the dominant triatomine bacterial symbiont in our study (59\% of the total $16 \mathrm{~S}$ coverage), but there were significant differences in the distribution of bacterial genera among vectors. In Rhodnius prolixus the dominant symbiont was Pectobacterium.
\end{abstract}

Conclusions: Trypanosoma cruzi-challenge significantly affects microbiota composition, with challenged vectors harbouring a significantly more diverse bacterial community, both in the gut and the gonads. Our results show that blood-feeding with T. cruzi epimastigotes strongly affects microbiota composition in a species-specific manner. We suggest that triatomine-adapted enterobacteria such as Arsenophonus could be used as stable vectors for genetic transformation of triatomine bugs and control of Chagas disease.

Keywords: Triatomine bugs, Chagas disease, Trypanosoma cruzi, Arsenophonus, Microbiota, Metabarcoding, 16 rRNA

\section{Background}

Microorganisms living in the midgut of insect vectors have an important role in modulating vector competence, which is the ability to acquire, maintain and transmit pathogens [1]. Microorganisms may interfere with vector competence either by direct interaction with parasites and competition for resources in the gut, or indirectly by inducing vector anti-parasitic activity and humoral immune

\footnotetext{
* Correspondence: karen.haag@ufrgs.br

'Programa de Pós-Graduação em Genética e Biologia Molecular,

Universidade Federal do Rio Grande do Sul, Porto Alegre, Rio Grande do Sul, Brazil

2Programa de Pós-Graduação em Biologia Celular e Molecular, Universidade Federal do Rio Grande do Sul, Porto Alegre, Rio Grande do Sul, Brazil Full list of author information is available at the end of the article
}

defence factors [2-5]. Furthermore, some symbiotic bacteria can be genetically modified to express anti-parasitic molecules or proteins that reduce insect fitness, as shown for Anopheles mosquitoes and Glossina tsetse flies in which transformed Pantoea and Sodalis symbionts were introduced, respectively [6, 7].

Triatomine bugs are vectors of the protozoan parasite $T$. cruzi, which causes Chagas disease. About 6 to 7 million people are estimated to be infected worldwide, mostly in Latin America where the disease is endemic [8]. In later years, T. cruzi became a public health concern also in the United States and other non-endemic countries, mostly due to human immigration from areas of endemicity [9-11]. It has been suggested that transmission might be controlled through the vector microbiota, 
since some strains of Serratia marcescens, a common symbiont of various triatomine species, have trypanolytic activity on several T. cruzi strains [12]. Methods for genetically modifying bacterial symbionts to effectively decrease parasite transmission or the development and fecundity of triatomine bugs have been established $[13,14]$.

The efficacy of such methods in the real world relies on the identification of key factors that influence the establishment of a successful T. cruzi infection on one hand, as well as the bacterial colonization of the insect gut on the other hand. A successful trypanosomatid infection can reduce or alter the triatomine gut microbiota composition [15-17]. Moreover, the ability of some $T$. cruzi strains to develop in certain vector species depends on the intrinsic qualities of either the parasite or the insect vector, as well as the resident host gut microbiota [18]. Previous studies using non-culturing based methods [19, 20] identified some triatomine gut microbiota characteristics: first, its diversity within each host is low with only one or few genera being dominant; secondly, some bacterial genera appear to be specific to certain triatomine hosts, i.e. Rhodococcus to Rhodnius and Arsenophonus to Triatoma; finally, lab-reared insects lose part of the original microbiota diversity but conserve most of the bacterial groups found in their wild counterparts.

Differently from other hemipterans, triatomine bugs do not possess specialized cells to harbour symbiotic bacteria (bacteriocytes). Most of the microbiota members have an extracellular lifestyle in the midgut lumen, being hypothetically acquired through the consumption of faeces of conspecifics (coprophagy) or by cannibalism [21]. Therefore, defining the key factors that influence triatomine gut microbiota composition remains a challenge, considering the numerous variables that need to be taken into consideration, such as the diversity of host species, symbiont mode of transmission and lifestyle, as well as the influence of $T$. cruzi infection, among other factors.

To evaluate the role of $T$. cruzi on the diversity and composition of triatomine microbiota, we metabarcoded the bacterial 16S rRNA gene of guts and gonads of colony-reared individuals belonging to six triatomine species, and compared insects that received a blood meal containing $T$. cruzi epimastigotes ( $T$. cruzi-challenged) with insects that were fed with parasite-free blood (nonchallenged).

\section{Methods}

\section{Insect sampling and dissection}

The six species of triatomine bugs analyzed in our study belong to three different genera, i.e., Panstrongylus megistus, Rhodnius prolixus, Triatoma infestans, T. brasiliensis, T. juazeirensis and T. sherlocki, with the last three species corresponding to the so called " $T$. brasiliensis complex" according to Costa et al. [22]. All insects from the same species were adults that came from a single colony. They have been maintained at the Laboratório de Doenças Parasitárias of Oswaldo Cruz Institute (Fiocruz, IOC) in Rio de Janeiro, and date from 1987 to 2011 depending on the species (Additional file 1: Table S1). A control group (unchallenged) was fed with $10 \mathrm{ml}$ citrated rabbit blood $(0.1 \mathrm{ml}$ sodium citrate/ $1 \mathrm{ml}$ blood). A second group (T. cruzi-challenged) was fed with $10 \mathrm{ml}$ of rabbit decomplemented blood containing T. cruzi epimastigotes. Decomplemented blood was obtained by an initial centrifugation at $3,500 \mathrm{rpm}$ for $10 \mathrm{~min}$ to separate the plasma from the erythrocytes. The plasma was discarded and red cells were washed in $3 \times$ phosphate-buffered saline (PBS), then resuspended in $10 \mathrm{ml}$ liver infusion tryptose (LIT) medium containing $1.5 \times 10^{7}$ epimastigotes in exponential growth phase per millilitre. We used the T. cruzi strain 0354, belonging to the Discrete Typing Unit I (DTU I), isolated from $T$. brasiliensis insects naturally infected from the municipality of Caicó ( $\mathrm{RN}$, Brazil). In addition, the gut microbiota of one adult P. megistus female collected in a peridomestic area in the municipality of Parobé (RS, Brazil), and positive for T. cruzi as confirmed by microscopy, was sampled as well. Dissections were performed for T. cruzi-challenged insects at day 10 after the challenge meal. Control and challenged insects were fed and dissected in parallel. Sterile ultrafine scissors and forceps were used to open the ventral side of specimens from the last thoracic segment to the last abdominal segment, and dissect the midgut (stomach and intestine) and gonads. The organs were separated in individual sterile tubes and washed twice with $1 \mathrm{ml} 1 \times$ PBS before DNA extraction. All procedures were performed under laboratory aseptic conditions.

\section{DNA extraction and sequencing}

DNA from gut and gonads was extracted using DNeasy Blood \& Tissue Kit (Qiagen, Hilden, Germany), according to the manufacturer's protocol, with a previous lysozyme treatment to break the cell walls of gram-positive bacteria. DNA concentrations were determined on a NanoDrop ND-1000 spectrophotometer (Thermo Fisher Scientific Inc., Waltham, Massachusetts, USA). The quality of bacterial DNA was verified using an initial amplification of the 16S rRNA gene, with primers Bakt_341F (5'-CCT ACG GGN GGC WGC AG-3') and Bakt_805R (5'-GAC TAC HVG GGT ATC TAA TCC-3') [23], which generate a fragment of 464 bp corresponding to the V3-V4 regions of the E. coli $16 \mathrm{~S}$ rRNA gene. In addition, detection of T. cruzi was performed by PCR of a $180 \mathrm{bp}$ fragment of the parasite satellite DNA using primers TcZ1 and TcZ2 [24]. For 16S rDNA amplicon 
sequencing, PCR was performed in triplicates, using modified versions of the original primers, in which an individual tag barcode of $8 \mathrm{nt}$ was added to the $5^{\prime}$ end (Additional file 1: Table S1). PCR reactions were performed in a total volume of $25 \mu \mathrm{l}$ containing $2 \mu \mathrm{l}$ of total DNA (200 ng on average), 1× PCR buffer, $1.5 \mathrm{mM}$ $\mathrm{MgCl}_{2}, 0.2 \mathrm{mM}$ of each dNTP, $1.25 \mathrm{U}$ of GoTaq DNA polymerase (Promega, Madison, WI, USA), and $0.4 \mathrm{mM}$ of each primer. DNA contamination was controlled by performing negative PCR reactions with $2 \mu \mathrm{l}$ of sterile water or with $2 \mu \mathrm{l}$ of the eluate of a DNeasy column that went through the kit protocol but no tissue was added. PCR included an initial denaturation at $95{ }^{\circ} \mathrm{C}$ for $5 \mathrm{~min}$, followed by 20 cycles of $94^{\circ} \mathrm{C}$ denaturation for $30 \mathrm{~s}, 55^{\circ}$ $\mathrm{C}$ annealing for $30 \mathrm{~s}$ (with a touchdown of $0.5{ }^{\circ} \mathrm{C}$ every cycle) and $72{ }^{\circ} \mathrm{C}$ extension for $20 \mathrm{~s}$, and another 15 cycles with an annealing temperature of $45{ }^{\circ} \mathrm{C}$, with a final extension step at $72{ }^{\circ} \mathrm{C}$ for $10 \mathrm{~min}$. PCR products were visualized by electrophoresis on $1.5 \%$ agarose gels, and the remaining amplicon volume was purified using PureLink PCR Purification Kit (Invitrogen, Carlsbad, CA, USA). The final products were pooled and $2 \times$ 300 paired-end sequenced on an Illumina Miseq flowcell using the Illumina MiSeq Reagent Kit Version3 (Illumina, San Diego, CA, USA) at Fasteris facilities (Geneva, Switzerland).

\section{Sequence processing and bacteria identification}

Sequencing reads were processed using Mothur v. 1.36.1 [25]. Forward and reverse paired end reads were merged and assigned to their respective replicate according to the tag barcodes. Sequences shorter than $250 \mathrm{bp}$, containing ambiguous bases, with homopolymer stretches longer than 15 bases or having mismatches in primer sequences, were discarded. Non-bacterial sequences were removed by performing a preliminary classification using the SILVA v119 $\mathrm{nr}$ database [26]. Chimeric sequences were removed with Mothur's implementation of UCHIME [27]. A final dataset was obtained in which singleton sequences as well as samples with a number of sequences equal or smaller than the highest number of sequences obtained for negative controls $(2,000$ sequences) were discarded. Finally, Operational Taxonomic Units (OTUs) were identified at $97 \%$ sequence similarity using the nearest neighbour option, and classified with a confidence threshold of $80 \%$ with the SILVA database ver. 119 [26]. Phylogenetic inferences were made to verify the relationships of our most abundant OTUs with other known bacteria. We searched for the most similar $16 \mathrm{~S}$ sequences to each OTU in the GenBank non-redundant database. The multiple alignment was made with MAFTT v. 7.187 [28], and phylogenetic analyses were performed with PhyML [29] as implemented in Geneious ver 8.1.5 (Biomatters, Auckland,
New Zealand) using the GTR + G + I model. Local support values were estimated by nonparametric bootstrap based on 100 re-samplings.

\section{Alpha and beta diversity analyses}

Rarefaction curves and alpha-diversity estimators, i.e. sample coverage (Good's coverage), richness (Chao1 index), and diversity (Shannon and Inverse Simpson index), were obtained with Mothur for each individual host. Mann-Whitney U-tests were used to evaluate the chance that a random sampling would result in differences between alpha-diversity means as far apart as those observed in our samples. To analyse the influence of host species, host gender, tissue and T. cruzi-challenge on bacterial community composition, we carried out a two-way Permutational Multivariate Analysis of Variance (PERMANOVA) of Bray-Curtis dissimilarities with Past 3.13 [30], in which these factors were tested as sources of variation in bacterial community composition. A Redundancy Analysis (RDA) was performed to discriminate the relative degree of influence of each of the above variables on microbiota composition using the vegan package [31] in R. As strongly skewed OTU distributions can bias the ordinations, we evaluated different transformation approaches in a Detrended Canonical Correspondence Analysis (DCA) to find one that meets the linearity assumption of RDA. Accordingly, we found that the Hellinger transformation was the best model of community composition, with the longest axis being equal to 2.28 . To qualitatively identify the environmental sources of variation that most significantly contribute to the variation in bacterial community composition, a Monte Carlo permutation test and a variance partitioning analysis were used based on the RDA output.

\section{Results}

\section{Characterization of triatomine bacterial communities}

Our work includes a dataset with 81 samples (46 T. cruzi-challenged and 35 non-challenged) plus one $T$. cruzi-infected field-collected $P$. megistus female that was used for comparison (Additional file 2: Table S2). Eight insects showed positive T. cruzi PCR at day 10 postchallenge, i.e. $4 T$. braziliensis, $2 T$. sherlocki and $2 P$. megistus (Additional file 2: Table S2; Fig. 2). The parasite was not detected in any tissue of $T$. cruzi-challenged $R$. prolixus, $T$. infestans or $T$. juazeirensis by diagnostic PCR. The $16 \mathrm{~S}$ rDNA amplicons of lab-reared insects yielded 1,534,192 sequences (mean coverage $\pm \mathrm{SD}$, $18,940 \pm 11,207$ per sample) that were binned into 824 OTUs (mean $\pm \mathrm{SD}, 85 \pm 37$ per sample; Additional file 3: Table S3). Good's coverage estimators are close to 1 (Additional file 2: Table S2), i.e. individual samples reflect very well the entire sampled population, and rarefaction curves, where some samples do not reach 
saturation, indicate an overestimation of low coverage OTUs (Additional file 4: Figure S1). To analyse the distribution of bacterial taxa in our samples we focused on the 11 OTUs belonging to five bacterial orders (GenBank accession numbers KX011883-KX011909) that reached at least $1 \%$ of the total coverage, and pool the remaining OTUs in a single group (Figs. 1, 2).

Arsenophonus is by far the predominant bacterium in our samples of lab-reared insects, amounting 59\% of the total 16S rDNA coverage of our study, and a surprising abundance of two Staphylococcus OTUs is found in some samples of different species (Figs. 1,2). Bacterial community composition in the parasite-free gut is defined by host taxa, i.e. in $R$. prolixus the dominant OTU is Pectobacterium while for the T. brasiliensis complex, Arsenophonus is dominant (Fig. 2). Direct comparison between the gut and gonads of the same host individual suggests that some groups have the capacity to move across tissues within the body cavity, with Arsenophonus showing a consistent presence both in gut and gonad samples. These observations are confirmed by PERMANOVA, which showed that the bacterial community composition of different vectors is significantly dissimilar, whereas for distinct tissues no significant dissimilarities are observed (see below).

\section{Phylogenetic and ecological relationships}

Some OTUs identified in our study such as Staphylococcus, Morganella and Clostridium, are found in diverse environments (Fig. 1), linking their presence in our samples to the colony room environment. Four genera were previously described as members of the triatomine gut microbiota, i.e. Arsenophonus, Pectobacterium, Rhodococcus and Dietzia (Fig. 1). The triatomine gut microbiota seems to be dominated by Enterobacteria. Arsenophonus, the predominant bacterium of our study, is mainly an insect-

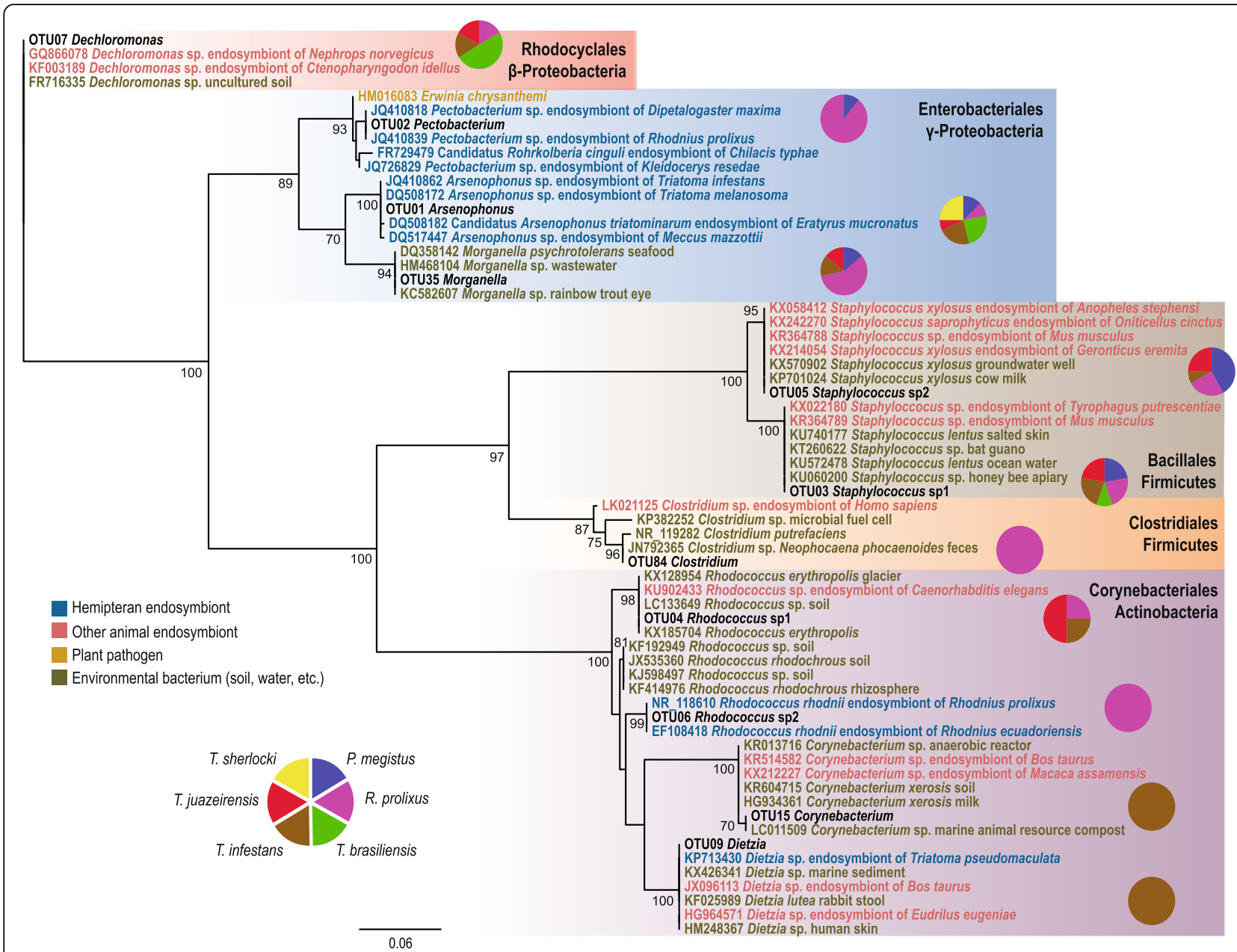

Fig. 1 Phylogenetic relationships of the 11 representative OTUs. The phylogeny includes the most closely related sequences of each OTU indicated by their GenBank accession numbers, and shown in different colours according to their origin. Pie charts display the frequency of samples (by host species) in which each OTU was found with at least $10 \%$ of the total coverage. Numbers at the internal nodes represent bootstrap support values ( $\geq 70 \%)$ 

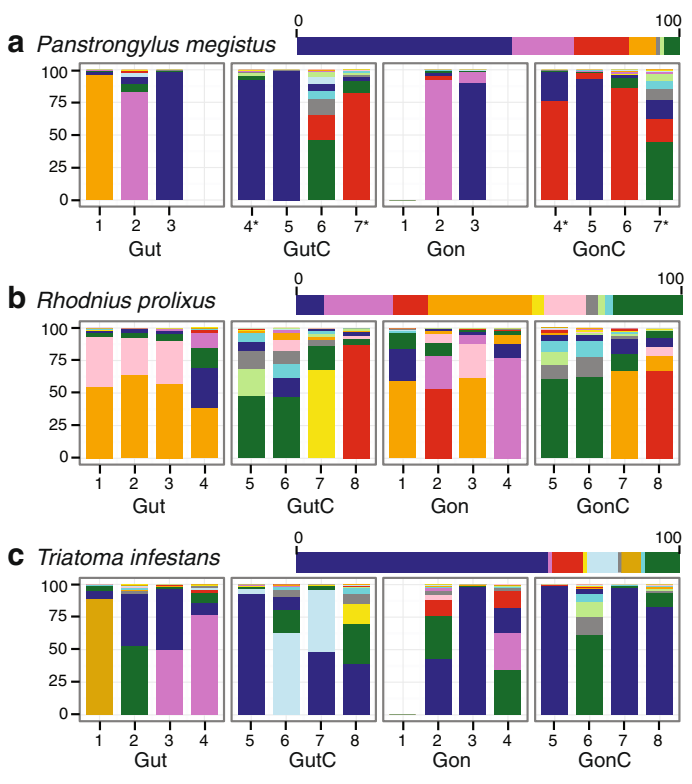

Arsenophonus (59\%)

Staphylococcus sp1 (8\%)

Staphylococcus sp2 (6\%)

Pectobacterium (6\%)

Rhodococcus sp1 (5\%)

Rhodococcus sp2 (2\%)

d T. brasiliensis

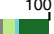

Dietzia (2\%)

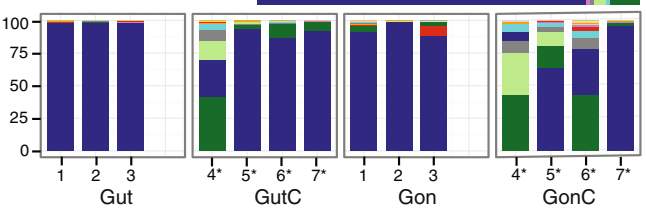

Morganella (1\%)

Decholoromonas (1\%)

Clostridium (1\%)

Corynebacterium (1\%)

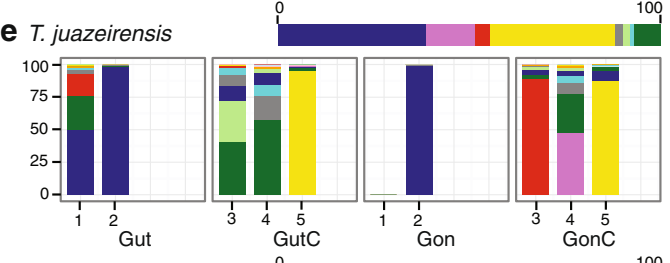

Other OTUs (8\%)

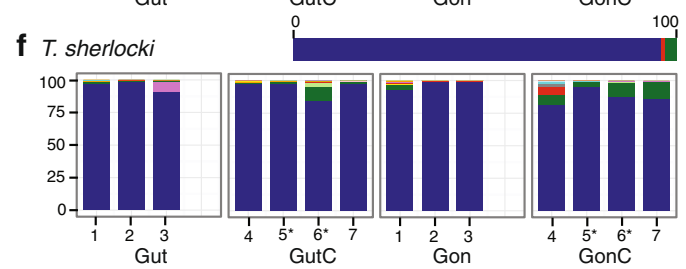

Fig. 2 Relative abundance of OTUs in the six species of triatomine bugs (a-f). Horizontal bars represent frequency estimates per species, and vertical bars, per sample. Abbreviations: Gut, gut samples of non-challenged insects; GutC, gut samples from T. cruzi-challenged insects; Gon, gonad samples of non-challenged insects; GonC, gonad samples from T. cruzi-challenged insects. Numbers identify unique individuals (see Additional file 2: Table S2 for details). Asterisks indicate individuals that showed positive T. cruzi infection at day 10 post-challenge

associated genus, including common triatomine endosymbionts (Fig. 1). Pectobacterium, another Enterobacteriales, was highly abundant $R$. prolixus, where Arsenophonus was less dominant (Fig. 2b). Similarly to Arsenophonus, the Pectobacterium OTU is closely related with other sequences isolated from triatomine bugs, i.e. R. prolixus and Dipetalogaster maximus (Fig. 1). For Rhodococcus, a Corynebacteriales, we identified two OTUs, i.e. Rhodococcus sp. 1 , which was found in $T$. cruzi-challenged $R$. prolixus and Triatoma spp. and Rhodococcus sp. 2, found mostly in non-challenged $R$. prolixus. These OTUs fall in separate clusters of our phylogeny (Fig. 1), with Rhodococcus sp. 1 being related to soil free-living bacteria, and Rhodococcus sp. 2 clustering with other Rhodnius endosymbionts. The latter is yet the only known Rhodococcus clade associated with insects. Corynebacterium and Dietzia, other Corynebacteriales, appeared in low frequency in our samples ( 1 and $2 \%$ of the total $16 \mathrm{~S}$ coverage, respectively, see Fig. 2).

Factors influencing the structure of bacterial communities Trypanosoma cruzi-challenged insects bear more diverse bacterial communities (Fig. 3a). The bacterial 


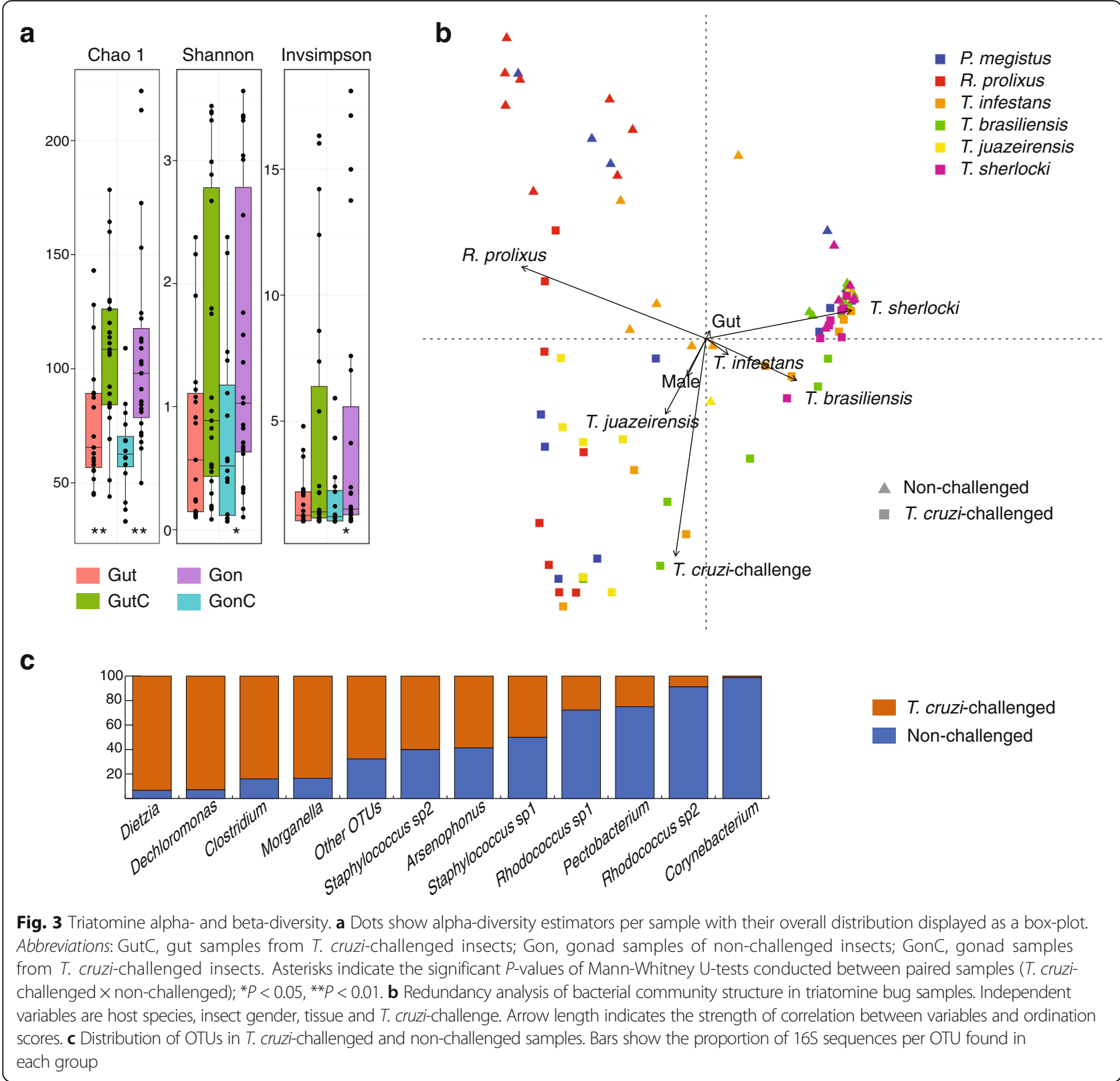

community of the T. cruzi-infected field-collected $P$. megistus female showed the highest alpha-diversity estimates, i.e. Chao1 index $=253.71$, Shannon index $=4.22$, and Inverse Simpson index $=25.56$ (Additional file 2: Table S2); Arsenophonus was the most abundant OTU (13.2\%; data not shown). To control for variations in their natural habitat, we studied the influence of $T$. cruzi-challenge on the microbiota of laboratory-reared vectors. Though not all experimentally challenged individuals established the infection after 10 days, $T$. cruzi-challenge seems to influence microbiota composition in a species-specific manner. A two-way PERMANOVA, in which host species and T. cruzi-challenge were defined as sources of variation of bacterial community composition suggests that both factors, as well as their interaction, contribute to the observed microbiota dissimilarities (Table 1). No significant contributions were detected for tissue or host gender.

To evaluate the relative impact of these factors in shaping microbiota structure in the triatomine bugs studied, an ordination analysis (RDA) was performed (Fig. 3b). The analysis showed that all four variables together explain $33.9 \%$ of total variance in the community composition (constrained variance). Monte Carlo permutation test $(n=199)$ confirmed that host species $(F=5.61, P=0.005)$ and $T$. cruzi-challenge $(F=7.07, P=$ $0.005)$ are the most significant variables. A variance partitioning of the four factors showed that host species 
Table 1 Two-way PERMANOVA of Bray-Curtis dissimilarities in triatomine microbiota composition

\begin{tabular}{|c|c|c|c|c|c|}
\hline Source of variation & Sum of squares & $d f$ & Mean square & $F$ & $P$ \\
\hline T. cruzi challenge & 0.4638 & 1 & 0.4638 & 2.1811 & 0.0353 \\
\hline Host species & 5.6166 & 5 & 1.1233 & 5.282 & 0.0001 \\
\hline Interaction & 0.2717 & 5 & 0.0543 & 0.2555 & 0.0003 \\
\hline Residual & 14.674 & 69 & 0.2126 & & \\
\hline Total & 21.026 & 80 & & & \\
\hline Host gender & 0.1024 & 1 & 0.1024 & 0.4201 & 0.7622 \\
\hline Host species & 5.6166 & 5 & 1.1233 & 4.6058 & 0.0001 \\
\hline Interaction & -1.5215 & 5 & -0.3043 & -1.2477 & 0.1937 \\
\hline Residual & 16.829 & 69 & 0.2439 & & \\
\hline Total & 21.026 & 80 & & & \\
\hline Tissue & 0.0732 & 1 & 0.0732 & 0.2968 & 0.8935 \\
\hline Host species & 5.6166 & 5 & 1.1233 & 4.5532 & 0.0001 \\
\hline Interaction & -1.6864 & 5 & -0.3372 & -1.3671 & 0.8263 \\
\hline Residual & 17.023 & 69 & 0.2467 & & \\
\hline Total & 21.026 & 80 & & & \\
\hline
\end{tabular}

Abbreviation: $d f$ degrees of freedom

and T. cruzi-challenge explain 25.9 and $6.7 \%$ of the total variance, respectively, while tissue (gut or gonad) and vector gender have a minimal influence, explaining less than $1 \%$ of the variance each.

\section{Discussion}

\section{Triatomine bacterial symbionts}

Under laboratory conditions, the triatomine gut microbiota is characterized by low diversity, being dominated by one or few bacterial genera. Some differences in the taxonomic composition of triatomine gut bacterial communities were found in our study, in comparison with previous results obtained with non-culture- $[19,20]$ and culture-based methods (reviewed in great extension by Vallejo et al. [32]). A significant absence in our samples is Serratia, a genus of bacteria that have trypanolytic activity on specific T. cruzi strains [12]. We discarded PCR bias in our experiments as an explanation for such discrepancy, because our primers perfectly match their respective annealing sites in all seven copies of the $16 \mathrm{~S}$ gene of the $S$. marcescens genome (Additional file 5: Figure S2). Furthermore, OTU160, one of the low frequency OTUs, with coverage of $25 \times$, was identified as Serratia (Additional file 3: Table S3). About half (12/25) of the Serratia sequences came from the field-collected $P$. megistus, suggesting that its association with the host might be dependent on environmental transmission in the natural habitat. Indeed, its loss under laboratory conditions has been previously described [20].

Interactions with intracellular symbionts that are vertically transmitted (P-symbionts [33]) should be more stable and therefore conserved across individuals of the same host species [34]. In our study, only the presence of Arsenophonus was consistent across all six triatomine species, but with varying degrees of dominance. All other bacteria identified in the microbiota of triatomine bugs in this study are clearly facultative (S-symbionts [33]). Most triatomine symbionts are probably extracellular and horizontally transmitted, being able to survive freely outside the host; environmental conditions such as $\mathrm{pH}$, oxygen levels and nutrient availability may act as filters allowing or not their colonization of the gut. Extracellular symbionts of hemipterans are horizontally transmitted by a multitude of routes, with coprophagy, cannibalism and environmental determination being the most prominent transmission strategies (see [34] for a review). The first studies on triatomine gut microbiota transmission hypothesized a horizontal route for the symbiont Rhodoccocus in $R$. prolixus via coprophagy of dry excreta [35].

A major exception seems to be Arsenophonus [36], which was highly abundant in the gonads of Triatoma spp. and P. megistus (Fig. 2), concordant with previous histological evidence [37]. Unlike other insects, where eggs become infected in early embryonic stages, Arsenophonus transmission in $T$. infestans occurs at some later stage, but nevertheless, vertical trans-ovarial transmission is confirmed by the presence of bacteria in the gut of the embryo prior to egg hatching [37]. Arsenophonus triatominarum, described by Hypša \& Dale [38] has now been identified in 17 species of triatomine bugs and represents the most numerous set of Arsenophonous lineages from closely related hosts [39]. Interestingly, aposymbiotic bugs derived by antibiotic treatment remain viable and capable 
of reproduction, and patterns of molecular evolution of $A$. triatominarum, which include genome degeneration, are thought to be typical of S-symbionts [40].

Pectobacterium (named in [19] as "Candidatus Rohrkolberia cinguli") was previously found in $R$. prolixus, $P$. megistus and Dipetalogaster maxima. The triatomine Pectobacterium symbiont is related to intracellular symbionts of Cimex lectularius (bedbug) [41] and Kleidocerys resedae (lygaeoid bugs) [42, 43]. In these groups, the bacterium is trans-ovarially transmitted and hosted in a bacteriome. Triatomine bugs do not develop specialized structures to harbor symbiotic bacteria; therefore, further studies are required to determine the habits and modes of transmission of Pectobacterium in the Triatominae. However, it is noteworthy that, since Arsenophonus and Pectobacterium are two core enterobacteria, they may both have similar habits and exploit the same transmission routes.

A second major bacterial order in the triatomine gut microbiota, the Corynebacteriales (Rhodococcus, Dietzia and Corynebacterium), includes extracellular facultative symbionts, common in diverse environments, notably in the soil ecosystem [44]. Rhodococcus was shown to be transmitted by coprophagy in Rhodnius spp. [35], suggesting that its presence in triatomine hosts should be more dependent on the environment, and maybe even random, in comparison to intracellular taxa. However, extracellular symbionts may exhibit similar mutual patterns of metabolic integration and even co-evolution with their hosts, as much as strictly intracellular symbionts [34], which could be the case of Rhodococcus-Rhodnius relationship.

\section{Microbiota interaction with Trypanosoma cruzi}

Over 60 of the approximate 148 species of triatomine bugs have been found naturally infected or have been experimentally infected with $T$. cruzi, suggesting that probably all species are potential vectors of the parasite $[45,46]$. However, different experimental combinations of triatomine species and/or parasite strains show that susceptibility is variable [47], with a tendency of local vectors to be more susceptible to parasite strains of $T$. cruzi from the same geographical areas [48]. In our experimental infection setting, we used a $T$. cruzi strain isolated from the parental population of our T. brasiliensis samples. It is therefore not surprising to find that most of the T. cruzi-positive samples, as detected by the diagnostic PCR, belong to this species. Two samples of closely related $T$. sherlocki, and the same number of $P$. megistus, were T. cruzi-positive as well.

We identified $T$. cruzi-challenge as the second most important factor shaping microbiota composition after host species. Such a pattern can be interpreted in the light of a tripartite vector-parasite-microbiota interaction (Fig. 4). After blood ingestion gut bacterial populations increase dramatically, probably due to iron and protein richness of the blood meal $[12,15]$, which in turn stimulates basal levels of insect immune activity (Fig. 4a). Key

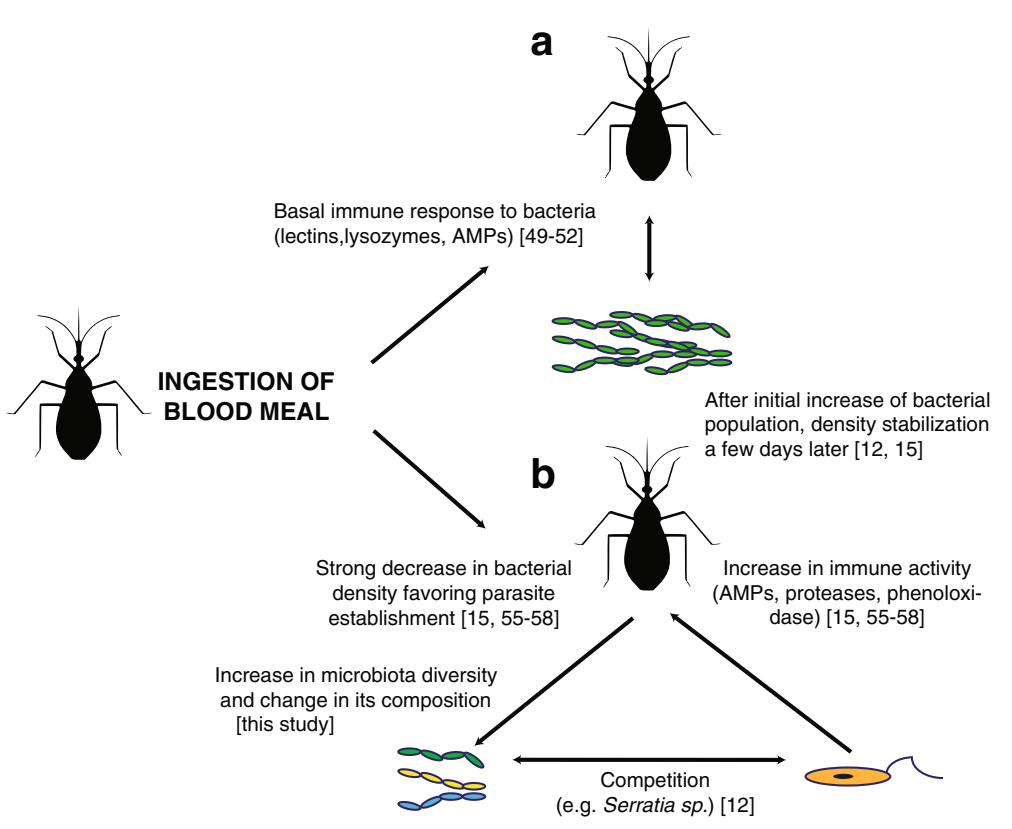

Fig. 4 Model of triatomine host-microbiota-parasite interactions based on parasite immune modulation. a Triatomine homeostatic responses to blood meal ingestion that control the density of bacteria in the gut. $\mathbf{b}$ Immune responses induced by $T$. cruzi infection control parasite development, and decrease bacteria density; intense competition for nutrients between bacteria and the protozoan occurs in the gut. An overall increase in micobiota diversity concomitant to infection is observed 
triatomine antimicrobial immunity includes recognition molecules such as lectins, lysozymes and antimicrobial peptides (AMPs, e.g. prolixicin and defensins, involved in the Imd immunological pathway) [49-54], responses that decrease bacterial load at approximately one week after feeding [15].

In our study, the microbiota was sampled 10 days post-epimastigote-artificial feeding, a period in which, in natural infection, trypomastigotes from ingested blood transform into replicative epimastigotes in the insect stomach, and in subsequent weeks they migrate to intestine and rectum $[18,55]$. This temporal window is not only important for parasite survival, but also for insect homeostasis. For establishing a successful infection, T. cruzi modulates vector immunity increasing the basal response against microbial proliferation after feeding, via an intensified activity of defensive routes including AMPs, phenoloxidases and antimicrobial proteases [15, 56-58] (Fig. 4b). In addition, some molecules that are found in the triatomine intestine such as agglutinant lectins and the pigment prodigiosin produced by the symbiont Serratia sp. can lyse some T. cruzi strains $[53,54]$. The intense competition between the parasite and the bacterial community, in addition to homeostatic microbiota-induced immune responses, leads to the ultimate increase in microbiota diversity (Figs. 3a, 4b), probably through the loss of some facultative symbionts and the invasion of more opportunistic bacteria (other OTUs in our study, see Fig. 2).

It is now known that $T$. cruzi reduces vector fitness $[59,60]$. In situations where the host is unable to eliminate the parasite via direct immune responses, it may have to rely on its microbiota to fight against the parasite. Indeed, it was shown for the bumblebeemicrobiota-trypanosomatid model system, that the microbiota drives both the general host defence against parasites and its specific interaction with different parasite genotypes [61]. In our study, the diagnostic PCR indicates that $T$. brasiliensis, $T$. sherlocki and $P$. megistus are susceptible to infection with $T$. cruzi strain 0354, and we have found Arsenophonus to be a dominant bacterium in the same three species. Contrary to Dietzia, Pectobacterium and Rhodococcus that are recognized members of the triatomine microbiota, but were found in our study either mostly in T. cruzi-challengend or unchallenged hosts, the prevalence of Arsenophonus does not seem to be affected by the parasite (Fig. 3c).

Exploring the tripartite host-microbiota-parasite model, one could predict that a mutualistic symbiotic relationship between bacteria and hosts would evolve in circumstances where the host benefits from decreasing the chance of being infected by the parasite, but parasites in turn should also evolve strategies to evade the defence conferred by symbiotic bacteria. Arsenophonus does not seem to be required for triatomine survival or reproduction [40] but it may have been allowed to live inside host cells and possibly being vertically transmitted due to some other benefit conferred to the host, e.g. defence against parasites or nutritional supplement.

\section{Conclusions}

Our study shows that there is a significant increase in diversity, and a change in composition, of the triatomine gut microbiota following the uptake of a T. cruzi-infected blood meal. Among all bacteria identified by $16 \mathrm{~S}$ rRNA gene metabarcoding in our study, Arsenophonus seems to be least susceptible to alterations caused by immune responses triggered by feeding, or by the presence of $T$. cruzi, possibly due to its intracellular lifestyle. In the last decade, different suggestions have been made regarding the use of gut microbiota for inhibiting the development of $T$. cruzi in triatomine bugs. We consider that the immune mechanisms used by the insect to control bacterial gut populations, the parasite response to these mechanisms and the functional role of symbionts, are critical to determine the effectiveness of these methodologies. Therefore, we suggest that Arsenophonus, with its intracellular lifestyle, is a good candidate for a stable paratransgenesis vector.

\section{Additional files}

Additional file 1: Table S1. Barcodes added to the $16 \mathrm{~S}$ forward (F) and reverse $(\mathrm{R})$ primers, identifying each amplicon sample. (XLSX $18 \mathrm{~kb}$ )

Additional file 2: Table S2. Triatomine sample information. (XLSX 19 kb) Additional file 3: Table S3. Bacterial OTU identification and coverage. (XLSX $43 \mathrm{~kb}$ )

Additional file 4: Figure S1. Rarefaction curves of samples per host species. (PDF $555 \mathrm{~kb}$ )

Additional file 5: Figure S2. Metabarcoding primers and their annealing sites on the 7 copies of the 16S rRNA gene of the Serratia marcescens genome (avalilable at http://www.ncbi.nlm.nih.gov/genome/ 1112). (PDF $929 \mathrm{~kb})$

\section{Abbreviations}

AMP: Antimicrobial Peptide; Imd: Immune deficiency pathway; LIT: Liver Infusion Tryptose

\section{Acknowledgments}

We are grateful to Sarah Urbano for her laboratory assistance, to Dr. José Artur Bogo Chies as well as his research group for providing us laboratory space, to Msc. Fernanda de Mello from FEEPPS/IPB-Lacen for providing us the field-collected sample, and to Dr. Angela Junqueira and Dr. Carlos José de Carvalho Moreira from Parasitic Diseases Laboratory (IOC/Fiocruz) for providing the insects used in this experiment. Finally, we also thank Dr. Carla Martins Lopes for introducing us to the world of metabarcoding.

\section{Funding}

Funding was provided by Fundação de Amparo à Pesquisa do Estado do Rio Grande do Sul (FAPERGS) grant number 1993-2551/13-1 and Conselho Nacional de Desenvolvimento Científico e Tecnológico (CNPq) grant number 470228/2013-0. 


\section{Availability of data and material}

The datasets supporting the conclusions of this article are included within the article and its additional files. The 16S rDNA sequences from the 11 representative OTUs identified in our study were deposited in GenBank with accession numbers KX011883-KX011909. The remaining data analysed during the current study are available from the corresponding author on reasonable request.

\section{Authors' contributions}

$\mathrm{SD}$ and KLH designed research; SD, BV, NC, JC and KLH performed the research; JC and KLH contributed new reagents/analytic tools; SD, BV and $\mathrm{KLH}$ analysed the data; SD, JC and KLH wrote the paper. All authors read and approved the final version of the manuscript.

\section{Competing interests}

The authors declare that they have no competing interests.

\section{Consent for publication}

Not applicable.

\section{Ethics approval and consent to participate}

Not applicable.

\begin{abstract}
Author details
'Programa de Pós-Graduação em Genética e Biologia Molecular, Universidade Federal do Rio Grande do Sul, Porto Alegre, Rio Grande do Sul, Brazil. ${ }^{2}$ Programa de Pós-Graduação em Biologia Celular e Molecular, Universidade Federal do Rio Grande do Sul, Porto Alegre, Rio Grande do Sul, Brazil. ${ }^{3}$ Laboratório de Biodiversidade Entomológica, Instituto Oswaldo Cruz-Fiocruz, Rio de Janeiro, Rio de Janeiro, Brazil. ${ }^{4}$ Departamento de Genética, Instituto de Biociências, Universidade Federal do Rio Grande do Sul, Porto Alegre, Rio Grande do Sul, Brazil.
\end{abstract}

Received: 30 September 2016 Accepted: 1 December 2016 Published online: 09 December 2016

\section{References}

1. Lane RS. Competence of ticks as vectors of microbial agents with an emphasis on Borrelia burgdorferi. In: Sonenshine DE, Mather TN, editors. Ecological Dynamics of Tick-borne Zoonoses. Oxford: Oxford University Press; 1994. p. 45-67

2. Azambuja P, Garcia ES, Ratcliffe NA. Gut microbiota and parasite transmission by insect vectors. Trends Parasitol. 2005:21:568-72.

3. Dillon RJ, Dillon VM. The gut bacteria of insects: nonpathogenic interactions Annu Rev Entomol. 2004:49:71-92.

4. Cirimotich CM, Ramirez JL, Dimopoulos G. Native microbiota shape insect vector competence for human pathogens. Cell Host Microbe. 2011:10:307-10.

5. Weiss B, Aksoy S. Microbiome influences on insect host vector competence. Trends Parasitol. 2011:27:514-22.

6. Wang S, Ghosh AK, Bongio N, Stebbings KA, Lampe DJ, Jacobs-Lorena M. Fighting malaria with engineered symbiotic bacteria from vector mosquitoes. Proc Natl Acad Sci USA. 2012;109:12734-9.

7. Aksoy S, Weiss B, Attardo G. Paratransgenesis applied for control of tsetse transmitted sleeping sickness. Adv Exp Med Biol. 2008:627:35-48.

8. World Health Organization (WHO). Chagas disease (American trypanosomiasis). Geneva: WHO; 2010

9. Montgomery SP, Starr MC, Cantey PT, Edwards MS, Meymandi SK. Neglected parasitic infections in the United States: Chagas disease. Am J Trop Med Hyg. 2014;90:814-8.

10. Bern C, Kjos S, Yabsley MJ, Montgomery SP. Trypanosoma cruzi and Chagas' disease in the United States. Clin Microbiol Rev. 2011;24:655-81.

11. Conners EE, Vinetz JM, Weeks JR, Brouwer KC. A global systematic review of Chagas disease prevalence among migrants. Acta Trop. 2016;156:68-78.

12. Azambuja P, Feder D, Garcia ES. Isolation of Serratia marcescens in the midgut of Rhodnius prolixus: Impact on the establishment of the parasite Trypanosoma cruzi in the vector. Exp Parasitol. 2004;107:89-96.

13. Taracena ML, Oliveira PL, Almendares O, Umaña C, Lowenberger C, Dotson EM, et al. Genetically modifying the Insect gut microbiota to control Chagas disease vectors through systemic RNAi. PLoS Negl Trop Dis. 2015;9:e0003358.
14. Ben BC, Cordon-Rosales C, Durvasula RV. Bacterial symbionts of the triatominae and their potential use in control of Chagas disease transmission. Annu Rev Entomol. 2002;47:123-41.

15. Castro DP, Moraes CS, Gonzalez MS, Ratcliffe NA, Azambuja P, Garcia ES Trypanosoma cruzi immune response modulation decreases microbiota in Rhodnius prolixus gut and is crucial for parasite survival and development. PLoS One. 2012;7:e36591.

16. Vieira CS, Mattos DP, Waniek PJ, Santangelo JM, Figueiredo MB, Gumiel M, et al. Rhodnius prolixus interaction with Trypanosoma rangeli: modulation of the immune system and microbiota population. Parasit Vectors. 2015;8:135.

17. Eichler S, Schaub GA. Development of symbionts in triatomine bugs and the effects of infections with trypanosomatids. Exp Parasitol. 2002;100:17-27.

18. Garcia ES, Ratcliffe NA, Whitten MM, Gonzalez MS, Azambuja P. Exploring the role of insect host factors in the dynamics of Trypanosoma cruziRhodnius prolixus interactions. J Insect Physiol. 2007:53:11-21.

19. da Mota FF, Marinho LP, de Moreira CJC, Lima MM, Mello CB, Garcia ES, et al. Cultivation-independent methods reveal differences among bacterial gut microbiota in triatomine vectors of Chagas disease. PLoS Negl Trop Dis. 2012;6:e1631.

20. Gumiel M, da Mota FF, de Sousa RV, Sarquis O, de Castro DP, Lima MM, et al. Characterization of the microbiota in the guts of Triatoma brasiliensis and Triatoma pseudomaculata infected by Trypanosoma cruzi in natural conditions using culture independent methods. Parasit Vectors. 2015;8:245.

21. Otálora-Luna F, Pérez-Sánchez AJ, Sandoval C, Aldana E. Evolution of hematophagous habit in Triatominae (Heteroptera: Reduviidae). Revista Chilena de Historia Natural. 2015;88:4

22. Costa J, Correia NC, Neiva VL, Gonçalves TCM, Felix M. Revalidation and redescription of Triatoma brasiliensis macromelasoma Galvão, 1956 and an identification key for the Triatoma brasiliensis complex (Hemiptera: Reduviidae: Triatominae). Mem Inst Oswaldo Cruz. 2013:108:785-9.

23. Herlemann DP, Labrenz M, Jürgens K, Bertilsson S, Waniek JJ, Andersson AF. Transitions in bacterial communities along the $2000 \mathrm{~km}$ salinity gradient of the Baltic Sea. ISME J. 2011;10:1571-9.

24. Russomando G, Figueredo A, Almiron M, Sakamoto M, Morita K. Polymerase chain reaction-based detection of Trypanosoma cruzi DNA in serum. J Clin Microbiol. 1992:30:2864-8.

25. Schloss PD, Westcott SL, Ryabin T, Hall JR, Hartmann M, Hollister EB, et al. Introducing Mothur: open-source, platform-independent, communitysupported software for describing and comparing microbial communities. Appl Environ Microbiol. 2009;75:7537-41.

26. Quast C, Pruesse E, Yilmaz P, Gerken J, Schweer T, Yarza P, et al. The SILVA ribosomal RNA gene database project: improved data processing and webbased tools. Nucleic Acids Res. 2013:41:D590-6.

27. Edgar RC, Haas BJ, Clemente JC, Quince C, Knight R. UCHIME improves sensitivity and speed of chimera detection. Bioinformatics. 2011:27:2194-200.

28. Katoh K, Standley DM. MAFFT multiple sequence alignment software version 7: Improvements in performance and usability. Mol Biol Evol. 2013; 30:772-80.

29. Guindon S, Gascuel O. A simple, fast, and accurate algorithm to estimate large phylogenies by maximum likelihood. Syst Biol. 2003;52:696-704.

30. Hammer $\varnothing$, Harper DAT, Ryan PD. PAST: paleontological statistics software package for education and data analysis. Paleontol Electronica. 2001;4:1-9.

31. Dixon P. VEGAN, a package of $R$ functions for community ecology. J Veg Sci. 2003;14:927-30.

32. Vallejo GA, Guhl F, Schaub GA. Triatominae-Trypanosoma cruzi/T. rangeli: Vector-parasite interactions. Acta Trop. 2009;110:137-47.

33. Moya A, Peretó J, Gil R, Latorre A. Learning how to live together: genomic insights into prokaryote-animal symbioses. Nat Rev Genet. 2008;9:218-29.

34. Salem H, Florez L, Gerardo N, Kaltenpoth M. An out-of-body experience: the extracellular dimension for the transmission of mutualistic bacteria in insects. Proc Biol Sci. 2015;282:20142957.

35. Brecher G, Wigglesworth VB. The transmission of Actinomyces rhodnii Erikson in Rhodnius prolixus Stål (Hemiptera) and its influence on the growth of the host. Parasitology. 1944:35:220-4.

36. Nováková E, Hypša V, Moran NA. Arsenophonus, an emerging clade of intracellular symbionts with a broad host distribution. BMC Microbiol. 2009:9:143.

37. Hypša V. Endocytobionts of Triatoma infestans: distribution and transmission. J Invertebr Pathol. 1993:61:32-8.

38. Hypša V, Dale C. In vitro culture and phylogenetic analysis of "Candidatus Arsenophonus triatominarum", an intracellular bacterium from the triatomine bug, Triatoma infestans. Int J Syst Bacteriol. 1997:47:1140-4. 
39. Šorfová P, Škeříková A, Hypša V. An effect of $16 S$ rRNA intercistronic variability on coevolutionary analysis in symbiotic bacteria: molecular phylogeny of Arsenophonus triatominarum. Syst Appl Microbiol. 2008;31:88-100.

40. Wilkes TE, Duron O, Darby AC, Hypša V, Nováková E, Hurst GDD. The genus Arsenophonus. In: Zchori-Fein E, Bourtzis K, editors. Manipulative Tenants: Bacteria Associated with Arthropods. Boca Raton: Taylor \& Francis; 2011. p. 225-44.

41. Hypša V, Aksoy S. Phylogenetic characterization of two transovarially transmitted endosymbionts of the bedbug Cimex lectularius (Heteroptera: Cimicidae). Insect Mol Biol. 1997;6:301-4.

42. Kuechler SM, Dettner K, Kehl S. Characterization of an obligate intracellular bacterium in the midgut epithelium of the bulrush bug Chilacis typhae (Heteroptera, Lygaeidae, Artheneinae). Applied and environmental microbiology. Am Soc Microbiol. 2011;77:2869-76.

43. Kuechler SM, Renz P, Dettner K, Kehl S. Diversity of symbiotic organs and bacterial endosymbionts of lygaeoid bugs of the families Blissidae and Lygaeidae (Hemiptera: Heteroptera: Lygaeoidea). Appl Environ Microbiol. 2011;77:2869-76

44. Goodfellow M, Jones AL. orynebacteriales ord. nov. In: Goodfellow M, Kampfer P, Busse H-J, Trujillo ME, Suzuki K-I, Ludwig W, et al., editors. Bergey's Manual of Systematic Bacteriology: Volume 5: The Actinobacteria. 2nd ed. New York: Springer; 2012. p. 235-43.

45. Lent H, Wygodzinsky P. Revision of the Triatominae (Hemiptera, Reduviidae), and their significance as vectors of Chagas' disease. Bull Am Mus Nat Hist. 1979;163:123-520.

46. Galvão C, Paula AS. Sistemática e evolução dos vetores. In: Galvão C, editor. Vetores da doença de Chagas. Curitiba: Sociedade Brasileira de Zoologia; 2015. p. 26-31.

47. Schaub GA. Interactions of trypanosomatids and triatomines. Adv Insect Phys. 2009;37:177-242

48. Perlowagora-Szumlewicz A, Muller CA, Moreira CJ. Studies in search of a suitable experimental insect model for xenodiagnosis of hosts with Chagas' disease: 4-The reflection of parasite stock in the responsiveness of different vector species to chronic infection with different Trypanosoma cruzi stocks. Rev Saude Publica. 1990;24:165-77.

49. Mesquita RD, Vionette-Amaral RJ, Lowenberger C, Rivera-Pomar R, Monteiro FA, Minx P, et al. Genome of Rhodnius prolixus, an insect vector of Chagas disease, reveals unique adaptations to hematophagy and parasite infection. Proc Natl Acad Sci USA. 2015;112:14936-41.

50. Araújo CAC, Waniek PJ, Stock P, Mayer C, Jansen AM, Schaub GA. Sequence characterization and expression patterns of defensin and lysozyme encoding genes from the gut of the reduviid bug Triatoma brasiliensis. Insect Biochem Mol Biol. 2006;36:547-60.

51. Ribeiro JMC, Genta FA, Sorgine MHF, Logullo R, Mesquita RD, Paiva-Silva GO, et al. An insight into the transcriptome of the digestive tract of the bloodsucking bug, Rhodnius prolixus. PLoS Negl Trop Dis. 2014;8:e2594.

52. Vieira CS, Waniek PJ, Mattos DP, Castro DP, Mello CB, Ratcliffe NA, et al. Humoral responses in Rhodnius prolixus: bacterial feeding induces differential patterns of antibacterial activity and enhances mRNA levels of antimicrobial peptides in the midgut. Parasit Vectors. 2014;7:232.

53. Garcia ES, Genta FA, de Azambuja P, Schaub GA. Interactions between intestinal compounds of triatomines and Trypanosoma cruzi. Trends Parasitol. 2010;26:499-505.

54. Garcia ES, Castro DP, Figueiredo MB, Azambuja P. Immune homeostasis to microorganisms in the guts of triatomines (Reduviidae): a review. Mem Inst Oswaldo Cruz. 2010;105:605-10.

55. Kollien A, Schaub G. The development of Trypanosoma cruzi in triatominae. Parasitol Today. 2000;16:381-7.

56. Vieira CS, Waniek PJ, Castro DP, Mattos DP, Moreira OC, Azambuja P. Impact of Trypanosoma cruzi on antimicrobial peptide gene expression and activity in the fat body and midgut of Rhodnius prolixus. Parasit Vectors. 2016;9:119.

57. Buarque DS, Gomes CM, Araújo RN, Pereira MH, Ferreira RC, Guarneri AA, et al. A new antimicrobial protein from the anterior midgut of Triatoma infestans mediates Trypanosoma cruzi establishment by controlling the microbiota. Biochimie. 2016;123:138-43.

58. Soares TS, Buarque DS, Queiroz BR, Gomes CM, Braz GR, Araujo RN. A kazaltype inhibitor is modulated by Trypanosoma cruzi to control microbiota inside the anterior midgut of Rhodnius prolixus. Biochimie. 2015;112:41-8.

59. Elliot SL, Rodrigues Jde O, Lorenzo MG, Martins-Filho OA, Guarneri AA. Trypanosoma cruzi, etiological agent of Chagas disease, is virulent to its triatomine vector Rhodnius prolixus in a temperature-dependent manner. PLoS Negl Trop Dis. 2015;9:e0003646.

60. Peterson JK, Graham AL, Dobson AP, Chávez OT. Rhodnius prolixus life history outcomes differ when infected with different Trypanosoma cruzi I strains. Am J Trop Med Hyg. 2015;93:564-72.

61. Koch H, Schmid-Hempel P. Gut microbiota instead of host genotype drive the specificity in the interaction of a natural host-parasite system. Ecol Lett. 2012;15:1095-103.

\section{Submit your next manuscript to BioMed Central and we will help you at every step:}

- We accept pre-submission inquiries

- Our selector tool helps you to find the most relevant journal

- We provide round the clock customer support

- Convenient online submission

- Thorough peer review

- Inclusion in PubMed and all major indexing services

- Maximum visibility for your research

Submit your manuscript at www.biomedcentral.com/submit
Biomed Central 